\title{
Simulation of mouthguard use in preventing dental injuries caused by different impacts in sports activities
}

\author{
João Paulo Mendes Tribst ${ }^{1}$ (C) $\cdot$ Amanda Maria de Oliveira Dal Piva ${ }^{1}$ (D) Alexandre Luiz Souto Borges ${ }^{1}$ (i) \\ Marco Antonio Bottino ${ }^{1}[$
}

Received: 29 May 2018 / Accepted: 1 August 2018 / Published online: 10 August 2018

(c) Springer-Verlag Italia S.r.l., part of Springer Nature 2018

\begin{abstract}
Purpose Due to recurring doubts by athletes regarding the effectiveness of mouthguards (MG), this study evaluated the stress distribution in the skull and teeth as a consequence of different impacts.

Methods Two human skulls were created using modeling software: one in maximum intercuspidal position and the other with a MG. Next, a boxing glove was allocated into each of the coordinate axes $(x, y$ and $z$ ) to represent a boxing punch $(1000 \mathrm{~N})$ : Jab $(x)$, Cross $(y)$ or Uppercut $(z)$. The six models were imported to the analysis software and the geometries were subdivided into a specific number of elements. The bonded contacts simulated a situation in which the athlete would not lose their MG during impact. The system constriction was in the foramen magnum region. The results were presented in stress maps and graphs.

Results A higher stress concentration in teeth was shown for the model without a MG under direct impact on the upper central incisors. According to von Mises stress, each punch resulted in stress being produced in distinct areas of the skull. The displacement was proportional to the Maximum Principal Stress result. MG use decreased the jaw movement relative to the maxilla and reduced the stress magnitude during impact in all situations.

Conclusions MG use is beneficial for all simulated impacts and assists in dampening the generated stresses. Direct impacts on tooth regions showed the higher stress values and the most efficacy in protection was evidenced when using a MG.
\end{abstract}

Keywords Dental trauma $\cdot$ Mouthguard $\cdot$ Sports dentistry $\cdot$ Craniomandibular complex $\cdot$ Finite element analysis $\cdot$ Shock absorption

Amanda Maria de Oliveira Dal Piva

amodalpiva@gmail.com

João Paulo Mendes Tribst

joao.tribst@ict.unesp.br

Alexandre Luiz Souto Borges

aleborges@ict.unesp.br

Marco Antonio Bottino

mmbottino@uol.com.br

1 Department of Dental Materials and Prosthodontics, São Paulo State University (Unesp), Institute of Science and Technology, Av. Eng. Francisco José Longo, No. 777. Jardim São Dimas, São José dos Campos, SP 12245-000, Brazil

\section{Introduction}

Basketball, football, hockey, martial arts, and boxing carry the highest risk of dental injuries [1]. Thus, the use of mouthguards during sports is a commonly encouraged procedure by dental surgeons and sports technicians [2,3]. Ninety percent $(90 \%)$ of facial injuries affect the maxillary region [4], putting the integrity of dental tissues at risk and causing severe trauma which can lead to tooth loss. Despite this, mouthguards can modify an athlete's breathing [5].

Mouthguard thickness and the choice of an appropriate elastic material during its manufacture is directly related to its capacity to absorb the impacts caused by accidents [6]. However, in situations where the impact does not directly affect the maxillo-mandibular region, there are still doubts about whether it really is beneficial to use a mouthguard regarding the mechanics of dissipating the injurious impact 
$[7,8]$. Therefore, mouthguard use aims to attenuate stresses in the facial region [9].

One of the methodologies used to verify generated stress is finite element analysis, in which a numerical computational simulation takes into account the geometry, elastic modulus and boundary conditions of specific situations and demonstrates the possible mechanical behavior of the system $[9,10]$. Despite the wide use of this mathematical method in simulating situations involving mouthguards, there are still no reports on the influence of different impact directions on the stress generated on an athlete who is using or not using a mouthguard. Thus, physical activity that involves different areas of possible impact may generate doubts about the benefit in using a mouthguard [11].

Therefore, the aim of this study was to analyze the influence of different impact regions on the skull in using or not using an individualized mouthguard through finite element method. In order to approximate a simulation of a real situation in which an athlete can receive impact to different areas of the face during a single activity, a boundary condition of three different boxing punches was selected. Each evaluated punch is located on a distinct axis from the Cartesian coordinate system in space. The present biomechanical study investigated the beneficial effect of using a mouthguard while being submitted to facial/head impacts in a sport where the mouthguard use is mandatory. To the scope, three different stress criteria were used. The hypotheses of the study were that there would be a benefit in using mouthguards independent of the impact region, and that there would be a difference in the stress magnitude values between the different simulated impacts.

\section{Methods}

The impact simulation herein was performed using the 3D finite element analysis methodology. For this, a skull model was created in computer aided design software (Rhinoceros 4.0-SR9 McNeil North America) based on the São Paulo State University database (Unesp). The upper and lower teeth were then shaped and inserted into the alveolus. Next, the final models (in a volumetric geometry) were replicated totaling two human skull models: one in the maximum intercuspidal position (clenched teeth/jaw, closed mouth) and the other with a $3 \mathrm{~mm}$ mouth opening (free functional space). The second model was created in a relaxed muscular position with the absence of occlusal contacts, and received an anatomical mouthguard with $4 \mathrm{~mm}$ thickness [12]. In order to represent an impacting object, a professional boxing glove was modeled following the manufacturer's dimensions (Boxing Gloves, Everlast Pro Style Training, $12 \mathrm{Oz}, 800 \mathrm{~g}$ ). The glove was allocated to each of the coordinate axes (Fig. 1) to represent a specific punch in a boxing match, and the value of $1000 \mathrm{~N}$ was assumed as unidirectional loading [13]. The load was applied on three different axes according to the evaluated punch: a Jab punch on the upper central incisor ( $X$ axis), a Cross punch on the zygomatic arch ( $Y$ axis) and an Uppercut punch on the mandibular symphysis ( $Z$ axis).

The models were imported into the computer-aided analysis software and complex geometries were subdivided into a specific number of finite elements based on the mesh convergence test $(10 \%)$ to reduce numerical errors during the results processing [14]. A bonded contact interface was used between all contacts, simulating a situation in which the athlete would not lose their mouthguard during impact. The system constriction was in the foramen magnum region, simulating insertion of the vertebral column, but allowing movement of the skull base to the sides and back during the test. Von Mises stresses (integration of all stress components into an equivalent value) were used as an indication for the stress energy generated during impact for qualitative analysis. Thus, tensile stress was analyzed as a failure criteria of friable structures such as simulated hard tissues [15]. Skull displacement was also analyzed in the different situations. Finally, the highest stress peak values (maximum principal stress from the upper incisors buccal face) in the teeth were plotted in scatter plots to better visualize the mouthguard's influence on the system's mechanical behavior. The mechanical properties used in the simulation are summarized in Table 1 [16-19].

\section{Results}

Stress can be visualized using a linear color scale where blue indicates the lowest stress values, and red represents the highest stress values. The results in the dental structures were determined by the maximal principal stress criteria, discriminating tensile areas and demonstrating higher concentration for the model not using mouthguard in the Jab punch situation (a direct impact on the upper central incisors), followed by an Uppercut punch (impact in the chin region), and finally a Cross punch in the zygomatic arch region (Fig. 2a).

Von Mises stress distributions in the models after impact are shown in Fig. 2b. The majority of stress in all models was in the dental structures, demonstrating that the ease of a trauma to affect these elements is greater than causing fracture in one of the facial bones. Each punch showed stress resulting in distinct areas of the skull. Thus, the displacement was proportional to the maximum principal stress result. The use of the mouthguard decreased the mandible movement relative to the maxilla in all simulated situations (Fig. 2c). The stress peaks, according to the picture of the dental arches (Fig. 2a), were plotted in a linear scatter plot 
Fig. $11000 \mathrm{~N}$ load application through different boxing punches

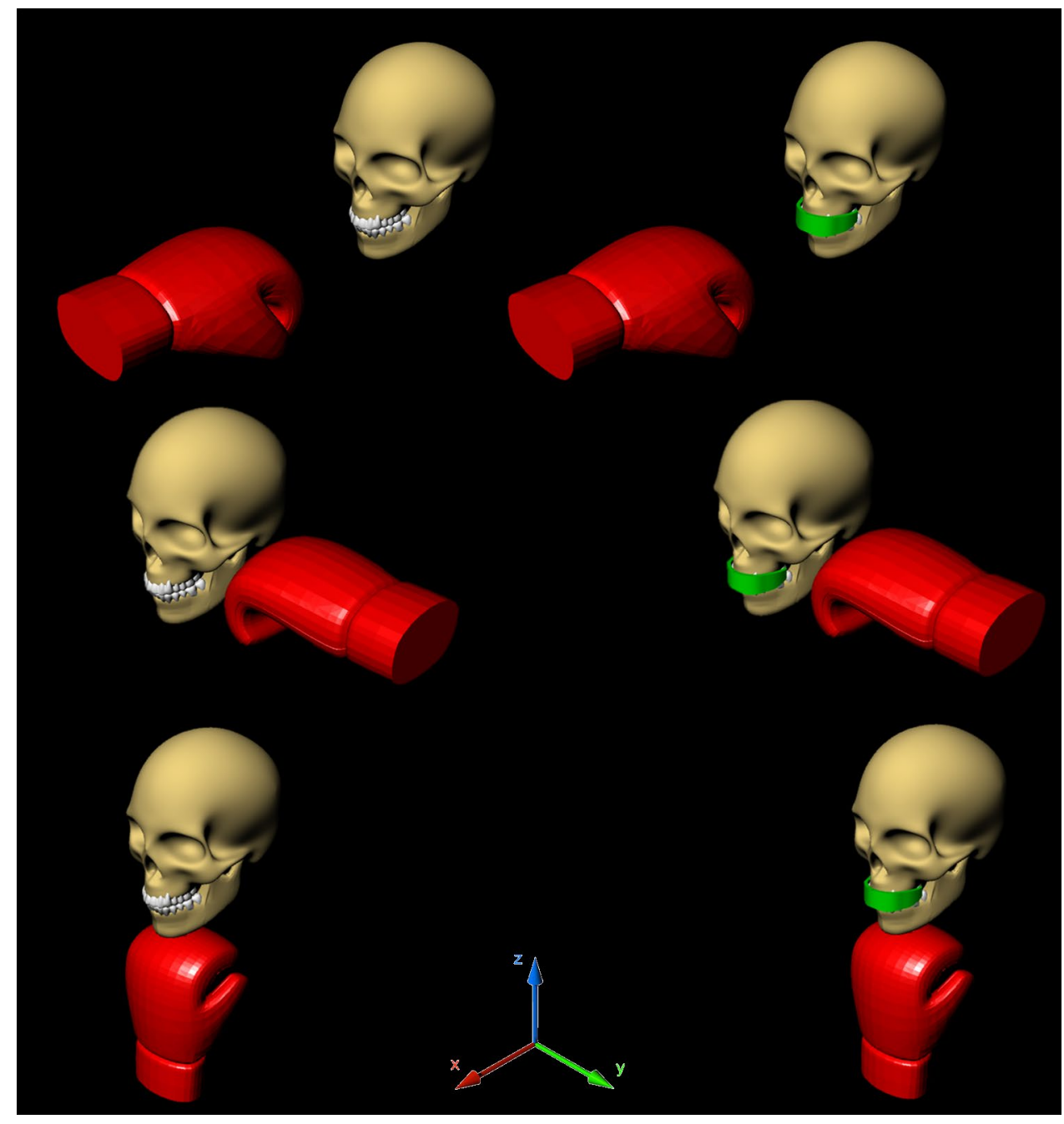

\section{Discussion}

Table 1 Mechanical properties of each material and structure used in the simulation

\begin{tabular}{lll}
\hline Material & $\begin{array}{l}\text { Elastic modulus } \\
(\mathrm{GPa})\end{array}$ & Poisson's ratio \\
\hline Mouthguard (EVA) [16] & 18 & 0.30 \\
Polyurethane [17] & 3.6 & 0.30 \\
Human bone tissue [18] & 13.7 & 0.3 \\
Human teeth [19] & $2.07 \times 10^{4}$ & 0.3 \\
\hline
\end{tabular}

for quantitative analysis (Fig. 3), and demonstrate that mouthguard use was significantly beneficial in all the simulated impacts. The higher tensile stress peaks (nodes 4 and 15 in Fig. 3) were observed in the cervical region of the right and left central upper incisors, respectively, during the Jab punch.
Several sports activities suggest using a mouthguard as an accessory for the athlete's individual protection $[2,3,8$, $11,13]$. However, the use of this apparatus is not unanimous among physical exercise practitioners, since doubt and acknowledgement for this preventive method have not been given due importance $[11,20]$. The results of this study show that there is a difference in stress distribution during physical activity as a function of the affected region and mouthguard use, thus accepting the hypothesis of this study. It is possible to observe that using a mouthguard is beneficial in all evaluated situations (Figs. 2, 3).

Using a mouthguard is more capable to reduce the stress when the impact region is nearer to the dental structures. Therefore, the further the impact region on the skull is from the mouth, the less influence the device has on stress dissipation. However, its use is essential and its benefits are not only restricted to directly preventing damage to the teeth. Thus, it is correct to assume that fracture cases 


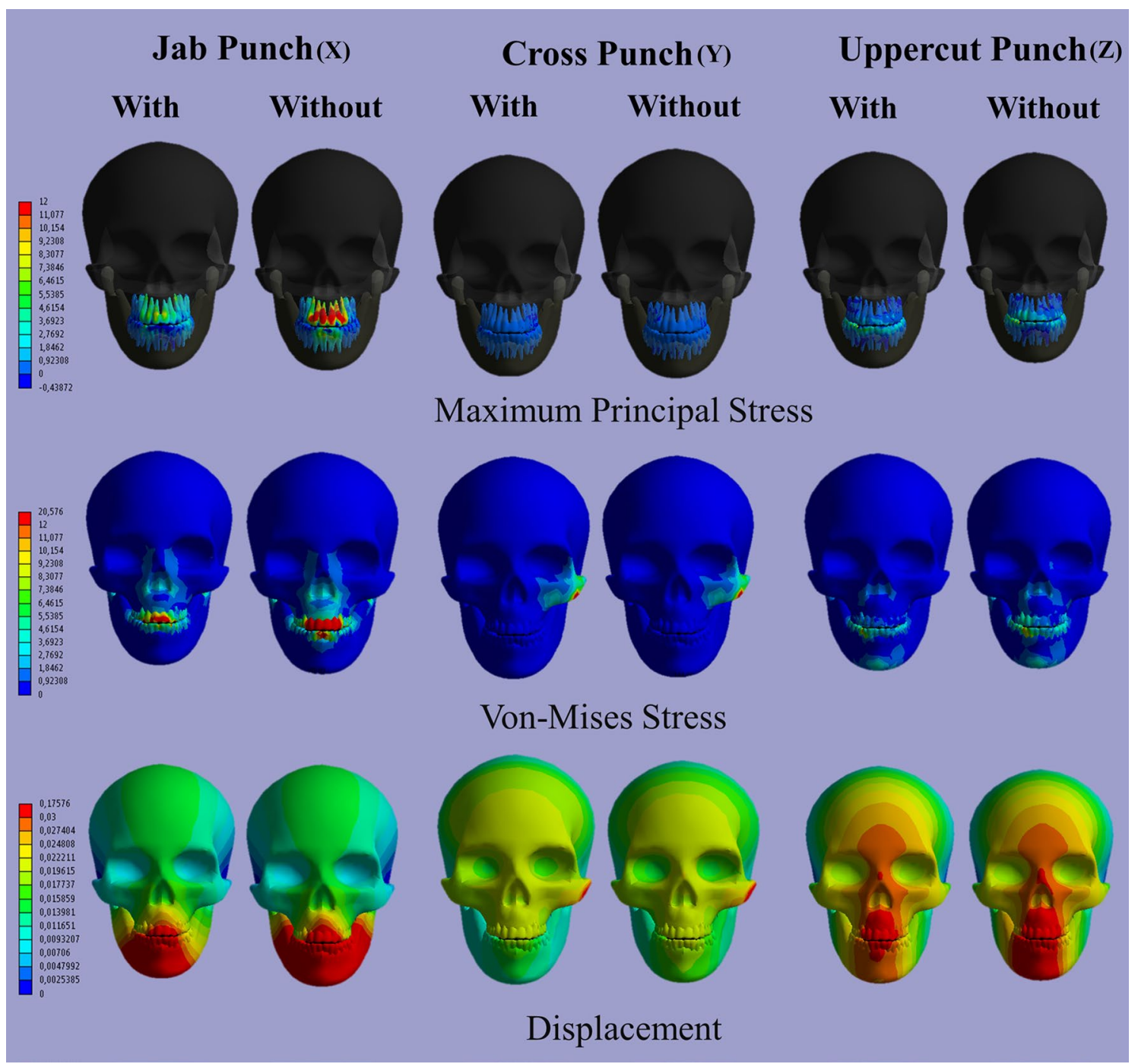

Fig. 2 The generated stress in the dental structures according to the punch and the stress criteria used

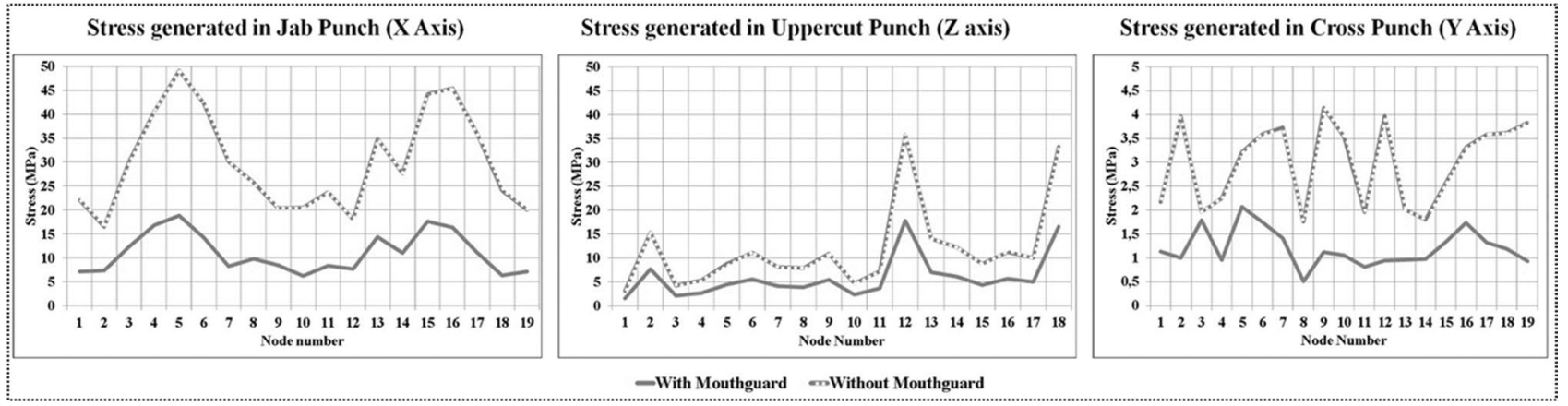

Fig. 3 Linear scatter plot of the stress peaks in each model with and without mouthguard according to the different boxing punches

could present worse prognosis if the athlete was not using a mouthguard [3, 8, 21-23]. It is hoped that doubts by athletes about the benefits of using a mouthguard diminish with this information, since this is one of the main reasons reported for this apparatus not being used during sports practice $[20,24]$. 
It is important to note that the simulated mouthguards had $4 \mathrm{~mm}$ thickness, which is described as an ideal preparation condition [12], so that thinner mouthguards may have different results [12]. In addition, the simulated mouthguard was customized, respecting the 3D model anatomy and closely related to the hard facial tissues. Thus, the results of the present study should be carefully extrapolated to risk situations that involve the use of stock or thermoplastic mouthguards [25].

Facial fractures were related to various sports which included baseball $(34.3 \%)$, rugby $(16.1 \%)$, soccer $(12.1 \%)$, softball $(8.9 \%)$, golf $(4.0 \%)$, hockey $(11.3 \%)$, and others (11.7\%) [26]. Despite this, a boxing simulation is not a methodology restriction, but a way to perform unidirectional loading on distinct axes from the spatial coordinates without escaping from the reality of a physical activity practitioner. According to the national collegiate athletic association's handbook (USA), mouthguard use is mandatory when athletes are participating in several sports, such as field hockey, football, ice hockey, and women's and men's lacrosse [27]. In addition, sports mouthguards are also mandatory in Canada for most minor hockey players, amateur football, amateur rugby, karate, taekwondo, lacrosse and amateur boxing. The only professional sport where sports mouthguards are mandatory is boxing [28].

Mechanical evaluation of mouthguards is generally performed with spherical objects fired at a controlled speed. This method is perfectly valid to study the variables of geometry and mouthguard material [7, 9, 10, 12, 25]. Nevertheless, since finite element analysis is a computational simulation in which a 3D model is defined in a design software, it is possible to simulate a complex condition with greater control over the environmental variables [9]. Thus, when analyzing damage generated during sports performance, we can guarantee that the impact will be accurate regarding force, speed, and direction, all controlled by the computer.

Von Mises stress enables a view of the total energy dissipation involved during impact on the face [12]. The compression and tensile zones result in a colorimetric map proportional to the exerted damage. In a frontal impact directed on the teeth, almost all stress is concentrated on the anterior dental elements. When using a mouthguard, the stress fields of the same region present less magnitude and concentration. As the fracture of the dental elements occurs by tensile stress [15], the maximum principal stress result only in the teeth allows us to observe that the roots are also affected upon the same type of impact, and the resulting mechanical von Mises stress behavior is similar. Finally, this can be explained by system displacement [14], since less energy will be dissipated along the skull when using the mouthguard (in occlusion, keeping the inferior teeth in contact with the device). The same interpretation is repeated for the impacts from an uppercut, and to a lesser extent for the cross punch.
Although using mouthguards is widely recommended and defined as an important way of preventing facial injuries [20], this device cannot overcome the damage generated to the face. Even when using mouthguards, all models herein presented impact stress in all structures. Despite the capacity of mouthguards to reduce stress during impact, some athletes report difficulties in breathing and speaking, nausea, oral dryness and some kind of uncomfortable perception [5, 29]. Also, the athletes' cardiopulmonary capacity can be negatively influenced when a stock mouthguard is used, while a custom-made device does not seem to have negative effects during its use [29]. Therefore, we simulated a custom-made mouthguard according to teeth anatomy. Future studies evaluating the biomechanical response according to the mouthguard design should be performed.

When a mouthguard is in the mouth, the displacement field between the mouthguard and buccal tissues can be considered continuous [9]. Thus, it was assumed that the mouthguard will not move during any of the applied forces. Moreover, removing the mouthguard could alter the protection capacity of the apparatus, dissipating part of the impact energy and resulting in different stress field regions [9, 10]. In this way, it is important to emphasize that the finite element method is a theoretical computational analysis. Simplifications were required in the 3D model for its performance such as absent musculature, soft tissues of the head and mouth, and the presence of anisotropic bone containing trabecular bone $[9,10]$. However, the results are important, and in association with the current literature can demonstrate the benefit of using a mouthguard, regardless of the impact on the head region, thus justifying the mandatory mouthguard use in some types of sports with appropriate interventions by sports federations. Our principal biomechanical novelty was to use three different stress criteria to show the beneficial effect of wearing a mouthguard in three facial/head impacts received while practicing a sport where its use is mandatory. Future three-dimensional investigations evaluating the effect of an impact in different faces (eg, broad and narrow), dental Classes, genders, ages, mouthguard design and material (with and without reinforcement), should be performed to obtain more detailed information regarding the mouthguard biomechanics. Furthermore, a detailed analysis of the soft tissues stresses should be added.

\section{Conclusions}

Within the limitations of this study, it is possible to conclude that using an individualized/customized mouthguard alleviates tensile stresses in the face, independent of the initial injury/impact site. Direct impacts on tooth regions showed the most aggressive stress values, and using a mouthguard (versus not) evidenced the most protective efficacy. 


\section{Compliance with ethical standards}

Conflict of interest The authors declare that they have no conflict of interest.

Ethical approval This article does not contain any studies with human participants or animals performed by any of the authors.

Informed consent For this type of study, formal consent is not required.

\section{References}

1. Young EJ, Macias CR, Stephens L (2015) Common dental injury management in athletes. Sports Health 7:250-255

2. Maeda Y, Kumamoto D, Yagi K, Ikebe K (2009) Effectiveness and fabrication of mouthguards. Dent Traumatol 25:556-564

3. Lloyd JD, Nakamura WS, Maeda Y, Takeda T, Leesungbok R, Lazarchik D et al (2017) Mouthguards and their use in sports: report of the 1st International Sports Dentistry Workshop, 2016. Dent Traumatol 33:421-426

4. Welch CL, Thomson WM, Kennedy R (2010) ACC claims for sports-related dental trauma from 1999 to 2008: a retrospective analysis. N Z Dent J 106:137-142

5. Kondo G (1997) Study on effects of mouth guard on ventilation. Kokubyo Gakkai Zasshi 64:326-347

6. Mizuhashi F, Koide K, Takahashi M (2013) Thickness and fit of mouthguards according to a vacuum-forming process. Dent Traumatol 29:307-312

7. Tanaka Y, Tsugawa T, Maeda Y (2017) Effect of mouthguards on impact to the craniomandibular complex. Dent Traumatol 33:51-56

8. Gialain IO, Kobayashi-Velasco S, Caldeira CL, Cavalcanti MGP (2017) Dental trauma prevention with mouthguard in a nose fracturing blow to the face: case report. Dent Traumatol 33:410-413

9. Otani T, Kobayashi M, Nozaki K, Gonda T, Maeda Y, Tanaka $M$ (2018) Influence of mouthguards and their palatal design on the stress-state of tooth-periodontal ligament-bone complex under static loading. Dent Traumatol. https://doi.org/10.1111/edt.12386 (Epub ahead of print)

10. Gialain IO, Coto NP, Driemeier L, Noritomi PY, Dias RB (2016) A three-dimensional finite element analysis of the sports mouthguard. Dent Traumatol 32:409-415

11. Galic T, Kuncic D, Poklepovic Pericic T, Galic I, Mihanovic F, Bozic J et al (2018) Knowledge and attitudes about sports-related dental injuries and mouthguard use in young athletes in four different contact sports: water polo, karate, taekwondo and handball. Dent Traumatol. https://doi.org/10.1111/edt.12394. (Epub ahead of print)

12. Verissimo C, Costa PV, Santos-Filho PC, Tantbirojn D, Versluis A, Soares CJ (2016) Custom-Fitted EVA Mouthguards: what is the ideal thickness? a dynamic finite element impact study. Dent Traumatol 32:95-102
13. Loturco I, Nakamura FY, Artioli GG, Kobal R, Kitamura K, Cal Abad CC et al (2016) Strength and power qualities are highly associated with punching impact in elite amateur boxers. J Strength Cond Res 30:109-116

14. Tribst JPM, Rodrigues VA, Borges ALS, Lima DR, Nishioka RS (2018) Validation of a simplified implant-retained cantilever fixed prosthesis. Implant Dent 27:49-55

15. Dal Piva AMO, Tribst JPM, Souza ROAE, Borges ALS (2017) Influence of alveolar bone loss and cement layer thickness on the biomechanical behavior of endodontically treated maxillary incisors: a 3-dimensional finite element analysis. J Endod 43:791-795

16. Peacock A (2000) Handbook of polyethylene: structures: properties, and applications. CRC Press, USA

17. Souza AC, Xavier TA, Platt JA, Borges AL (2015) Effect of base and inlay restorative material on the stress distribution and fracture resistance of weakened premolars. Oper Dent 40:E158-E166

18. Tanne K, Hiraga J, Kakiuchi K, Yamagata Y, Sakuda M (1989) Biomechanical effect of anteriorly directed extraoral forces on the craniofacial complex: a study using the finite element method. Am J Orthod Dentofacial Orthop 95:200-207

19. Tanne K, Hiraga J, Sakuda M (1989) Effects of directions of maxillary forces on biomechanical changes in craniofacial complex. Eur J Orthod 11:382-391

20. Liew AK, Abdullah D, Wan Noorina WA, Khoo S (2014) Factors associated with mouthguard use and discontinuation among rugby players in Malaysia. Dent Traumatol 30:461-467

21. Hino S, Takeda A, Kaneko T, Horie N, Shimoyama T (2017) Oral lacerations during motocross: a case report. Dent Traumatol 33:482-484

22. Rattai J, Levin L (2018) Oral injuries related to Ice Hockey in the province of Alberta, Canada: trends over the last 15 years. Dent Traumatol 34:107-113

23. Bergman L, Milardović Ortolan S, Žarković D, Viskić J, Jokić D, Mehulić K (2017) Prevalence of dental trauma and use of mouthguards in professional handball players. Dent Traumatol 33:199-204

24. Afrashtehfar KI, Chung J (2017) Mouthguard use may reduce dentofacial injuries in field hockey players. Evid Based Dent 18:48-49

25. Bochnig MS, Oh MJ, Nagel T, Ziegler F, Jost-Brinkmann PG (2017) Comparison of the shock absorption capacities of different mouthguards. Dent Traumatol 33:205-213

26. Yamamoto K, Matsusue Y, Horita S, Murakami K, Sugiura T, Kirita T (2018) Trends and characteristics of maxillofacial fractures sustained during sports activities in Japan. Dent Traumatol. https://doi.org/10.1111/edt.12395. (Epub ahead of print)

27. Mills SC (2015). Mandatory mouthguard rules for high school athletes in the United States. Gen Dent 63(6):35-40

28. Canadian Dental Hygienists Association (2005) CDHA position paper on sports mouthguards. Can JDent Hyg 39(6):1-18

29. Caneppele TMF, Borges AB, Pereira DM, Fagundes AA, Fidalgo TK, Maia LC (2017) Mouthguard use and cardiopulmonary capacity-a systematic review and meta-analysis. Sports Med Int Open 1(05):E172-E182 